\section{Infection Prevention Control Bundle of Multidrug-Resistant Acinetobacter baumannii and Methicillin-Resistant Staphylococcus aureus: Which One Is More Important?}

To the Editor-Infection prevention control (IPC) bundles are often employed as standard of care practices to minimize transmission of multidrug-resistant (MDR) microorganisms. ${ }^{1,2}$ Dedicated resource and leadership commitment are recognized assets in implementation of IPC bundles. Specific IPC interventions were previously reported in association with transmission of MDR Acinetobacter baumannii and methicillin-resistant Staphylococcus aureus (MRSA) in Thailand; multifaceted interventions were uncommon. ${ }^{3}$ We now report results of a national survey conducted to evaluate the IPC interventions associated with incidence of MDR A. baumannii and MRSA 12-months after implementation of IPC bundles. From January 1, 2012, through April 30, 2013, telephone interviews were conducted with the lead infection control professionals at 204 hospitals that previously participated in a survey to identify compliance to an IPC program and the incidence of MDR A. baumannii and MRSA infection before and after implementation of the IPC bundles.

The IPC interventions reported in this study included contact isolation, patient cohorts, hand hygiene campaigns, active surveillance, environmental cleaning, existence of antimicrobial stewardship programs (ASPs), use of chlorhexidine gluconate bathing, and use of hydrogen peroxide vaporizer. The
MDR A. baumannii IPC bundle A included contact isolation, patient cohorting, a hand hygiene campaign, environmental cleaning, and ASP. The IPC bundle B included bundle A plus active enteric surveillance for $A$. baumannii. The MRSA IPC bundle $C$ included contact isolation, patient cohorting, a hand hygiene campaign, active nasal surveillance, nasal decolonization with mupirocin, and ASP; the IPC bundle D included bundle $C$ plus chlorhexidine gluconate bathing. A $50 \%$ reduction in the mean incidence of MDR $A$. baumannii or MRSA infection compared with the 12-month preintervention period was defined as statistically significant. Data on the incidence of each of these pathogen groups and compliance with each IPC bundle component were obtained from each hospital's infection control database. Analyses were performed using SPSS, version 15 (SPSS). All $P$ values were 2tailed; $P<.05$ was considered to be statistically significant. Multivariate logistic regression models, stratified according to compliance, were performed to determine factors associated with a reduction in MDR A. baumannii and MRSA infection incidence.

Survey responses were obtained from representatives of all 204 hospitals (100\%) that participated in an earlier study. ${ }^{3}$ Overall, 184 hospitals (90.2\%) reported 1 or more IPC interventions targeting MDR $A$. baumannii, whereas 100 hospitals (49\%) reported 1 or more IPC interventions to control MRSA. For MDR A. baumannii, the most common IPC interventions were contact isolation (184 [90.2\%] of 204 hospitals) and a hand hygiene campaign (177 [86.8\%] of 204); $36(17.6 \%)$ of 204 reported use of IPC bundle A, and 36 (17.6\%) of 204 reported use of IPC bundle B for MDR $A$. baumannit; compliance ranged from $25 \%$ to $96 \%$ for all IPC

TABLE 1. Infection Prevention Control (IPC) Interventions Associated with Reduction in Multidrug-Resistant (MDR) Acinetobacter baumannii and Methicillin-Resistant Staphylococcus aureus (MRSA) Incidence as Reported by Representatives from 204 Thai Hospitals

\begin{tabular}{|c|c|}
\hline Pathogen, compliance, \% & aOR $(95 \% \mathrm{CI})$, by intervention \\
\hline \multicolumn{2}{|l|}{ MDR A. baumannii } \\
\hline $40-60$ & $\ldots$ \\
\hline $60-80$ & IPC bundle A: 1.55 (1.05-3.45); IPC bundle B: 1.69 (1.19-4.96) \\
\hline $80-100$ & $\begin{array}{l}\text { Hand hygiene: } 1.59 \text { (1.12-5.46); antimicrobial stewardship program: } 1.24 \text { (1.09-6.45); } \\
\text { IPC bundle A: } 2.45(1.41-6.93) \text {; IPC bundle B: } 2.93(1.56-5.69)\end{array}$ \\
\hline \multicolumn{2}{|l|}{ MRSA } \\
\hline $40-60$ & ... \\
\hline $60-80$ & IPC bundle D: $1.45(1.08-5.45)$ \\
\hline $80-100$ & $\begin{array}{l}\text { Hand hygiene: } 1.55 \text { (1.06-4.93); contact isolation: } 1.05 \text { (1.01-5.46); IPC bundle D: } \\
\quad 3.36(2.12-5.69)\end{array}$ \\
\hline
\end{tabular}


components. For MRSA, the 2 most common IPC interventions were hand hygiene campaigns (100 [49\%] of 204) and contact isolation (84 [41.2\%] of 204); $18(8.8 \%)$ of 204 reported use of IPC bundle C, and $10(4.9 \%)$ of 204 reported use of IPC bundle D. Compliance ranged from $20 \%$ to $94 \%$ for all IPC components. By multivariate analysis, no significant reduction in MDR A. baumannii or MRSA infection was evident among hospitals with less than $60 \%$ IPC compliance. Over the 1-year period, there were significant reductions in MDR A. baumannii among hospitals with $60 \%-$ $80 \%$ compliance to IPC bundles $A$ and $B$ and significant reduction in MRSA among hospitals with $60 \%-80 \%$ compliance to IPC bundle D (Table 1). Having greater than $80 \%$ compliance with hand hygiene, contact isolation, ASP, and IPC bundles were associated with reduction in MDR A. baumannii and MRSA infection.

Our study findings emphasize the need for multifaceted interventions featuring a "horizontal" approach to control the spread of MDR A. baumannii and MRSA. ${ }^{4,5}$ We acknowledge that the report of these survey findings includes limitations of sample size and recall biases related to survey design, execution, and analysis. Despite such limitations, we have identified modifiable gaps and opportunities for implementation of IPC bundles to limit transmission of MDR A. baumannii and MRSA in resource-limited settings.

\section{ACKNOWLEDGMENTS}

Financial support. This study was supported by the National Research University Project of the Thailand Office of Higher Education Commission (to A.A.).

Potential conflicts of interest. L.M.M. reports that she is an employee of GlaxoSmithKline (GSK) and that this work was conducted pro bono and independently of GSK. A.A. declares no conflicts of interest relevant to this article. All authors submitted the ICMJE Form for Disclosure of Potential Conflicts of Interest, and the conflicts that the editors consider relevant to this article are disclosed here.

Anucha Apisarnthanarak, $M{ }^{1}$ Linda M. Mundy, $\mathrm{MD}, \mathbf{P h D}^{2}$

Affiliations: 1. Division of Infectious Diseases, Faculty of Medicine, Thammasat University Hospital, Pathumthani, Thailand; 2. GlaxoSmithKline, Bryn Mawr, Pennsylvania.

Address correspondence to Anucha Apisarnthanarak, MD, Division of Infectious Diseases, Faculty of Medicine, Thammasat University Hospital, Pathumthani, Thailand 12120 (anapisarn@yahoo.com).

Infect Control Hosp Epidemiol 2014;35(2):207-208

(C) 2014 by The Society for Healthcare Epidemiology of America. All rights reserved. 0899-823X/2014/3502-0020\$15.00. DOI: 10.1086/674865

\section{RE F E R E N C E S}

1. World Health Organization. Prevention and containment of antimicrobial resistance. http://www.searo.who.int/LinkFiles /BCT_Reports_SEA-HLM-408.pdf. Accessed April 1, 2011.

2. Apisarnthanarak A, Pinitchai U, Thongphubeth $K$, et al. A mul- tifaceted intervention to reduce pandrug-resistant Acinetobacter baumannii colonization and infection in 3 intensive care units in a Thai tertiary care center: a 3-year study. Clin Infect Dis 2008; 47:760-767.

3. Apisarnthanarak A, Khawcharoenporn T, Mundy LM. Practices to prevent multidrug-resistant Acinetobacter baumannii and methicillin-resistant Staphylococcus aureus in Thailand: a national survey. Am J Infect Control 2013;41:416-421.

4. Wenzel RP, Edmond MB. Infection control: the case for horizontal rather than the vertical intervention programs. Int $J$ Infect Dis 2010;14(suppl 4):S3-S5.

5. Edmond MB, Wenzel RP. Screening inpatients for MRSA: case closed. New Engl J Med 2013;368:2314-2315.

\section{Germ and Hematology: Underlying Disease Influences Diversity of Germ Spectra and Antibiotic Therapy}

To the Editor-Knowledge of microbiological germ spectra is a crucial prerequisite for calculated and empirical antibiotic therapy, especially for immunocompromised patients. The microbiological spectra of hematology patients, irrespective of the isolation site, may differ from that of other patients, and taking this into consideration may substantially influence the choice of antibiotics at admission, especially in an outpatient setting or in emergency departments. To elucidate the potential variability of microbiological spectra, we analyzed all consecutive admissions of patients with infectious complications at the Medical Center of the Otto-von-Guericke University in Magdeburg, Germany, over an 18-year period from January 1992 through December 2009. In this retrospective, single-center study, the microbiological isolates obtained from collected patient samples from the Department of Hematology and Oncology (hematology department patients [HP], who were mostly patients with acute leukemia and lymphoma) were compared with those obtained from all other departments (non-hematology department patients $[\mathrm{NHP}]$, who were treated in the medical and surgical departments).

Within the relevant period, 603,944 pathogens were isolated, of which 21,431 (3.5\%) were obtained from HP. When comparing HP with NHP, the most frequent isolates were derived from blood cultures $(43.2 \%$ vs $15.8 \% ; P<.001)$ with an overall predominance of gram-positive bacteria. In HP specimens, the proportion of gram-positive bacteria was significantly higher than in NHP specimens $(67.4 \%$ vs $58.4 \%$; $P<.001)$. Anaerobic bacteria were found less frequently in HP samples than in NHP samples $(0.6 \%$ vs $1.0 \% ; P=.02)$. No difference was detectable between the groups with respect to yeasts. Staphylococcus aureus, Enterobacteriaceae, and Pseudomonadaceae were significantly less frequent among $\mathrm{HP}$ than among NHP, whereas the prevalence of coagulase-negative 